\title{
Cardiac-Specific Overexpression of miR- 222 Induces Heart Failure and Inhibits Autophagy in Mice
}

\author{
Ming Su ${ }^{\mathrm{a}}$ Zhiguo Chen ${ }^{\mathrm{a}}$ Changxin Wang ${ }^{\mathrm{a}}$ Lei Song ${ }^{\mathrm{a}}$ Yubao Zou ${ }^{\mathrm{a}}$ \\ Lianfeng Zhang ${ }^{b}$ Rutai Huia Jizheng Wanga

\begin{abstract}
aState Key Laboratory of Cardiovascular Disease, Fuwai Hospital, National Center for Cardiovascular Disease, Chinese Academy of Medical Sciences and Peking Union Medical College, Beijing, ${ }^{b} K e y$ Laboratory of Human Diseases Comparative Medicine, Ministry of Health, Institute of Laboratory Animal Science, Chinese Academy of Medical Sciences and Peking Union Medical College, Beijing, China
\end{abstract}

\section{Key Words}

miR-222 • Heart failure $•$ Autophagy $\bullet$ mTOR

\begin{abstract}
Background: MicroRNAs play a crucial role in the regulation of pathological cardiac remodeling and heart failure. Previously, we found that overexpression of miR-221 induces heart failure in mice. The $m i R-222$ and $m i R-221$ share the same gene cluster, however, the role of miR-222 in the regulation of cardiac function remained ill-defined. Methods and Results: Transgenic mice with cardiac-specific expression of miR-222 (Tg-miR-222) mice were generated. The Tg-miR-222 mice developed significantly enlarged hearts at 4 weeks of age. Transthoracic echocardiograph data indicated that the hearts of Tg-miR-222 mice exhibited an increased left ventricular end-diastolic internal diameter and decreased fractional shortening. We observed that the LC3-II in Tg-miR-222 mice was decreased accompanied with the upregulation of p62, indicating the autophagy inhibition in the hearts of Tg-miR-222 mice. The mTOR pathway, a negative regulator of autophagy, was activated in the hearts of $\mathrm{Tg}-\mathrm{miR}-222$ mice. The expression of p27 was downregulated by miR-222 overexpression. Conclusion: Our data indicate that miR-222 overexpression induces heart failure in mice. The downregulation of p27 and the activation of mTOR pathway may be involved in miR-222-induced heart failure and autophagy inhibition. Thus, targeting miR-222 expression may be a therapeutic strategy against pathological cardiac remodeling.

\section{Introduction}

Heart failure is the terminal outcome of many cardiovascular diseases, and it severely impacts the quality of life of affected patients. MicroRNAs (miRNAs) are small, non-coding

M. Su and Z. Chen contributed equally.

Jizheng Wang, Ph.D.

and Rutai Hui, M.D., Ph.D.

KARGER
State Key Laboratory of Cardiovascular Disease, Fuwai Hospital, National Center for

Cardiovascular Disease, Chinese Academy of Medical Sciences and Peking Union

Medical College, Beijing 100037 (China)

E-Mail wangjizheng@fuwaihospital.org / huirutai@gmail.com 


\section{Cellular Physiology Cell Physiol Biochem 2016;39:1503-1511

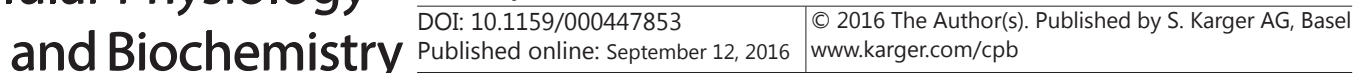 Su et al.: miR-222 Induces Heart Failure}

regulatory molecules that can play a critical role in heart failure [1]. Previously, we found that miR-221 is a pro-hypertrophic miRNA, both in vitro and in vivo $[2,3]$. The hearts of miR-221 cardiac-specific transgenic mice showed pathological cardiac hypertrophy and severe heart failure [3]. Further investigation indicated that miR-221-induced cardiac remodeling is associated with the downregulation of p27, activation of the mTOR pathway, and the subsequent inhibition of autophagy in cardiomyocytes [3]. The p27 gene is robustly expressed in adult cardiomyocytes, and it plays an anti-hypertrophic role in response to adverse stimulations [4-6]. Furthermore, p27 was also found to protect cardiomyocytes from apoptosis by inducing autophagy during metabolic stress [7]. These data highlight that p27 is a key negative regulator of pathological cardiac remodeling.

Human miR-222 and miR-221 encoding genes are located on the $\mathrm{X}$ chromosome and share the same gene cluster. Additionally, the sequences of these two mature miRNAs are similar, and their binding sites are identically conserved. Therefore, the target genes of these two miRNAs closely coincide. The p27 gene is a bona fide target of the miR-221/ miR-222 cluster [8-13]. In exercise-induced cardiac growth and acute heart injury models, the expression of miR-222 is upregulated and promotes cardiomyocyte proliferation [14]. Therefore, we hypothesize that cardiac-specific overexpression of miR-222 might impair normal cardiac function by targeting p27. In the present study, we investigated whether miR-222 showed similar effects as miR-221 in the heart. By constructing transgenic mice with cardiac-specific expression of miR-222 (Tg-miR-222), we found that Tg-miR-222 mice experience severe heart failure.

\section{Materials and Methods}

\section{Animal studies}

All of the animal studies were approved by the Ethics Committee for Animal Study of Fuwai Hospital (Beijing, China). The protocol for the construction of Tg-miR-222 mice was followed as previously described [3]. Briefly, the encoding gene of the miR-222 precursor was amplified by using the following primers: forward, 5'- GCG AGC TCG GCG GGG AGA GGG T-3'; reverse, 5'- CGC CGA AGC TTA GAT GAT ACA TAG ACA GCT GGA TAG-3'. The PCR product was digested with SacI and HindIII (TaKaRa, Dalian, China), and cloned into a pBSII- $\alpha$-MHC-hGH vector driven by the murine $\alpha$-MHC promoter. The constructs were then digested with NotI to linearize the plasmid. The products were injected into the pronucleus of fertilized zygotes from C57BL/6J mice, and then transferred to the oviducts of pseudopregnant ICR recipients. The genotypes of all the transgenic mice were verified by PCR analysis using tail genomic DNA and the following primers: $5^{\prime}$ GAC AGC AGA TCA CGA TTC TCC CG-3' and 5'-CTG AGG GCA CCC TAA TCC TTC TGA GAG TTA GAC A- $3^{\prime}$. The Fabpi gene was amplified as a control, using the following primers: forward, $5^{\prime}$-TGG ACA GGA CTG GAC CTC TGC TTT CCT AG A-3'; reverse, 5'-TAG AGC TTT GCC ACA TCA CAG GTC ATT CAG-3'. At 4 weeks of age, male transgenic mice were used for further analysis.

\section{Transthoracic echocardiography}

Transthoracic echocardiography analysis was performed as previously described [15]. In brief, the mice at both 4 and 16 weeks of age were weighed and anesthetized using $2.5 \%$ Avertin $(0.018 \mathrm{ml} / \mathrm{g})$. The two-dimensional short and long axes of the left ventricular (LV) were detected using a 30-MHz probe interfaced with a Vevo-770 high-frequency ultrasound system (VisualSonics, Toronto, ON, Canada). The LV end-diastolic internal diameter (LVIDd), LV end-systolic internal diameter (LVIDs), LV posterior wall thickness at the end of diastole (LVPWd) and LV anterior wall thickness at the end of diastole (LVAWd) were detected by using M-mode recordings. The fractional shortening (FS) measurements were calculated with the following formula: FS\% $=($ LVIDd - LVIDs $) /$ LVIDd $\times 100 \%$.

\section{Morphological analysis}

The hearts were fixed with 4\% paraformaldehyde, dehydrated, and embedded in paraffin. The paraffin sections were stained with Masson's trichrome reagents for histological analysis. The collagen deposition 


\section{Cellular Physiology Cell Physiol Biochem 2016;39:1503-1511

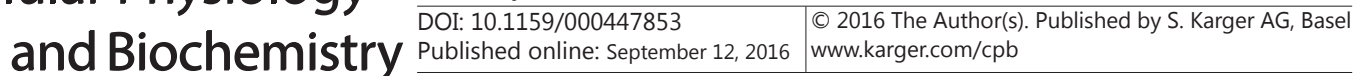 \\ Su et al.: miR-222 Induces Heart Failure}

was measured with Image-Pro Plus 6.0 software (Media Cybernetics Inc., Bethesda, Maryland, MD, USA). The collagen deposition (\%) was calculated with fibrotic area/total area $\times 100 \%$ in each field, and at least 5 random fields were observed for each mouse.

\section{Terminal deoxynucleotidyl transferase-mediated dUTP nick-end labeling staining}

The terminal deoxynucleotidyl transferase dUTP nick-end labeling (TUNEL) assay was performed using the in situ cell death detection kit, POD (Roche Diagnostics GmbH, Germany), according to the manufacturer's protocol. Briefly, tissue sections were de-paraffinized and rehydrated. The endogenous peroxidase was blocked with $3 \% \mathrm{H}_{2} \mathrm{O}_{2}$ for $30 \mathrm{~min}$. Next, the sections were digested with Proteinase $\mathrm{K}$ for $30 \mathrm{~min}$ at room temperature, and then incubated with TdT enzyme containing digoxigenin-dUTP working solution. The reaction was stopped, washed, and subsequently incubated with a POD working solution. The peroxidase activity was detected by adding diaminobenzidine. The sections were then stained with hematoxylin. The images in each section were obtained with an Olympus IX71 microscope (Tokyo, Japan). The TUNEL ${ }^{+}$and total nuclei were counted blind to mouse genotype. The apoptosis was calculated as TUNEL $^{+}$nuclei/total nuclei $\times 100 \%$, and at least 5 random fields were measured for each mouse.

\section{Cell culture and transfection}

Primary neonatal rat cardiomyocytes (NRCM) were isolated and cultured as previously described [2]. In brief, hearts from neonatal Wistar rats were isolated and minced. The heart tissues were digested with $0.06 \%$ collagenase (Worthington Biochemical Corporation, Lakewood, NJ, USA) at $37^{\circ} \mathrm{C}$. The cells were subjected to differential adhesion and plated in DMEM medium supplemented with 10\% FBS and $0.1 \mathrm{mM}$ bromodeoxyuridine. The cells were then plated and acclimated for 24 before treatment.

The cells were transfected with the miRNA mimic using Lipofectamine 2000 transfection reagent (Life Technologies), according to manufacturer's protocol. The final concentration of the miRNA mimic was 100 nM.

\section{RNA analysis}

Total RNA was extracted and isolated by using a miRNeasy Mini Kit (Qiagen, Hilden, Germany). The expression of miR-222 and miR-221 in the hearts of NTG and Tg-miR-222 mice was detected by quantitative RT-PCR. U6 was employed as the loading control. The relative expression was expressed as the $\Delta \mathrm{Ct}$ (compressive tracking) value. For the quantitative RT-PCR assay, complementary DNAs (cDNAs) were synthesized using a cDNA synthesis kit (TaKaRa, Dalian, China). The following specific primers were used: mouse atrial natriuretic peptide (ANP), 5'-AGT GCG GTG TCC AAC ACA G-3' and 5'-TGC TTC CTC AGT CTG CTC ACT C-3'; mouse brain natriuretic peptide (BNP), $5^{\prime}$-CTT TAT CTG TCA CCG CTG GGA G- $3^{\prime}$ and $5^{\prime}$-TTT GGG TGT TCT TTT GTG AGG C-3'; mouse GAPDH, 5' -GGC ATT GTG GAA GGG CTC-3' and $5^{\prime}$-GGG GGT AGG AAC ACG GAA G-3'.

\section{Protein analysis}

Proteins were extracted and quantified. A total of 20-50 $\mu \mathrm{g}$ of protein was loaded onto SDS polyacrylamide gels for electrophoretic separation. Then, the proteins were transferred onto a nitrocellulose membrane and blocked with 5\% non-fat dry milk at room temperature for $1 \mathrm{~h}$. The following primary antibodies were used and diluted to 1:1000 in TBST buffer for incubation: p27, p62, and GAPDH (Cell Signaling Technology, Beverly, MA, USA); the rabbit anti-LC3B antibody was purchased from Sigma-Aldrich (St. Louis., MO, USA). The membrane was then washed and incubated with HRP-conjugated secondary antibody for $1 \mathrm{~h}$ at room temperature. The bands were detected with SuperSignal West Femto Maximum Sensitivity Substrate (Pierce, Rockford, IL, USA). The intensity of each band was quantified by using Quantity One software, V4.6.2 (Bio-Rad, Hercules, CA, USA).

\section{Statistical analysis}

Statistical analysis was performed with SPSS 19.0 software (SPSS Inc., Chicago, IL, USA). A twotailed Student's t-test was used to determine the statistical significance between two groups. $\mathrm{P}<0.05$ was considered statistically significant. The data are presented as the mean \pm S.D. 


\section{Cellular Physiology Cell Physiol Biochem 2016;39:1503-1511

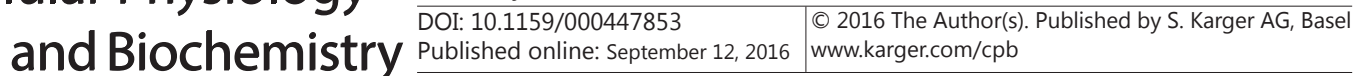 Su et al.: miR-222 Induces Heart Failure}

\section{Results}

Cardiac-specific overexpression of miR-222 induces heart failure in transgenic mice

To investigate the effect of miR-222 on the heart in vivo, we constructed transgenic mice with cardiac-specific expression of miR-222. The expression of miR-222 in the hearts of Tg-miR-222 mice was significantly higher than in their non-transgenic (NTG) littermates. The expression of miR-221, however, showed no difference between the two groups (Fig. 1A). The hearts of the Tg-miR-222 mice were significantly enlarged compared with the NTG mice (Fig. 1B). A histological analysis indicated that the ventricle chamber was significantly enlarged (Fig. 1C). The heart-to-body and lung-to-body ratios were both increased in TgmiR-222 mice (Fig. 1D and E), indicating that overexpression of miR-222 could result in heart failure. Accordingly, the expression of ANP and BNP in Tg-miR-222 mice was 18.6- and 5.8-fold higher than that of NTG mice, respectively (Fig. 1F).

We then detected the cardiac function in mice at both 4 and 16 weeks of age using high-resolution echocardiography (Fig. 2A). We found that the LVIDd was increased in TgmiR-222 mice; however, the LVAWd and LVPWd showed no differences between NTG and Tg-miR-222 mice at 4 and 16 weeks of age (Fig. 2B-I). The FS\%, a parameter that reflects cardiac function, was significantly decreased in Tg-miR-222 mice compared with their NTG littermates (Fig. 2E). These data indicated that overexpression of miR-222 led to pathological cardiac remodeling and impaired the cardiac function in vivo.

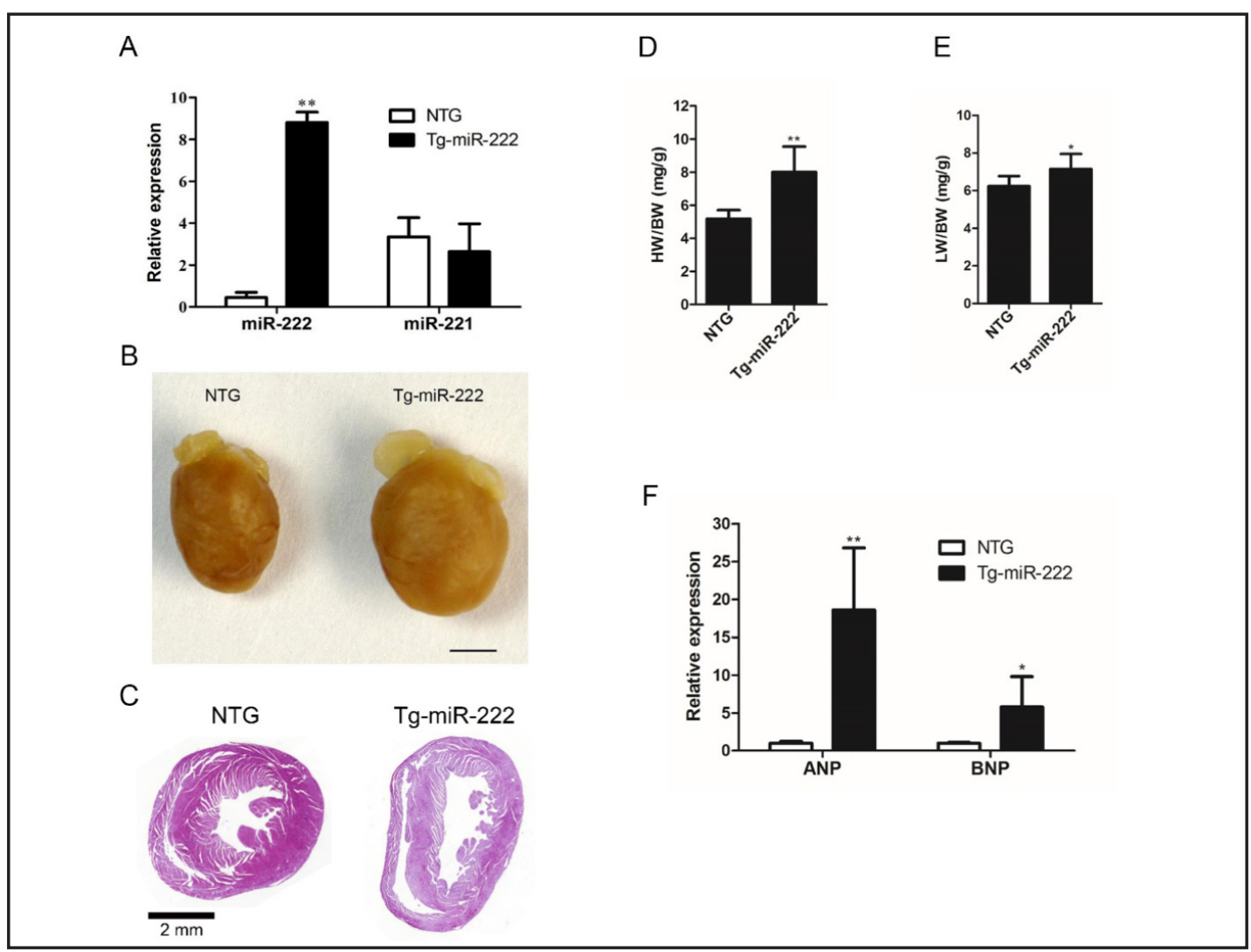

Fig. 1. Cardiac-specific overexpression of miR-222 induces pathological cardiac remodeling. (A) The relative expression of miR-222 and miR-221 in the hearts of miR-222 transgenic mice (Tg-miR-222) and the non-transgenic controls (NTG). (B) The morphology of hearts from Tg-miR-222 and NTG mice at 4 weeks of age. Scale bar: $2 \mathrm{~mm}$. (C) Hematoxylin/eosin staining of the hearts from NTG and Tg-miR-221 mice. Scale bar: $2 \mathrm{~mm}$. (D) Ratios of heart weight to body weight (HW/BW) in NTG and Tg-miR-222 mice (n=8 per group). (E) Ratios of lung weight to body weight (LW/BW) in NTG and Tg-miR-222 mice (n=8 per group). (F) The expression of ANP and BNP in hearts from NTG and Tg-miR-222 mice ( $n=8$ per group). 
Fig. 2. Cardiac-specific overexpression of miR-222 impairs heart function. (A) Representative M-mode echocardiography images. (B-I) Echocardiography parameters in mice at 4 (B-E) and 16 (F-I) weeks of age. The left ventricular internal dimension at diastole (LVIDd) (B, F), left ventricular anterior wall at diastole (LVAWd) $(C, G)$, left ventricular posterior wall at diastole (LVPWd) (D, H) and fractional shortening (FS\%) (E, I) were detected with a Vevo-770 high-frequency ultrasound system. The data are expressed as the mean \pm S.D. of NTG control mice $(n=8)$ and Tg-miR-222 mice $(\mathrm{n}=7) .{ }^{* *} \mathrm{P}<0.01$ compared with NTG mice of the same week of age.

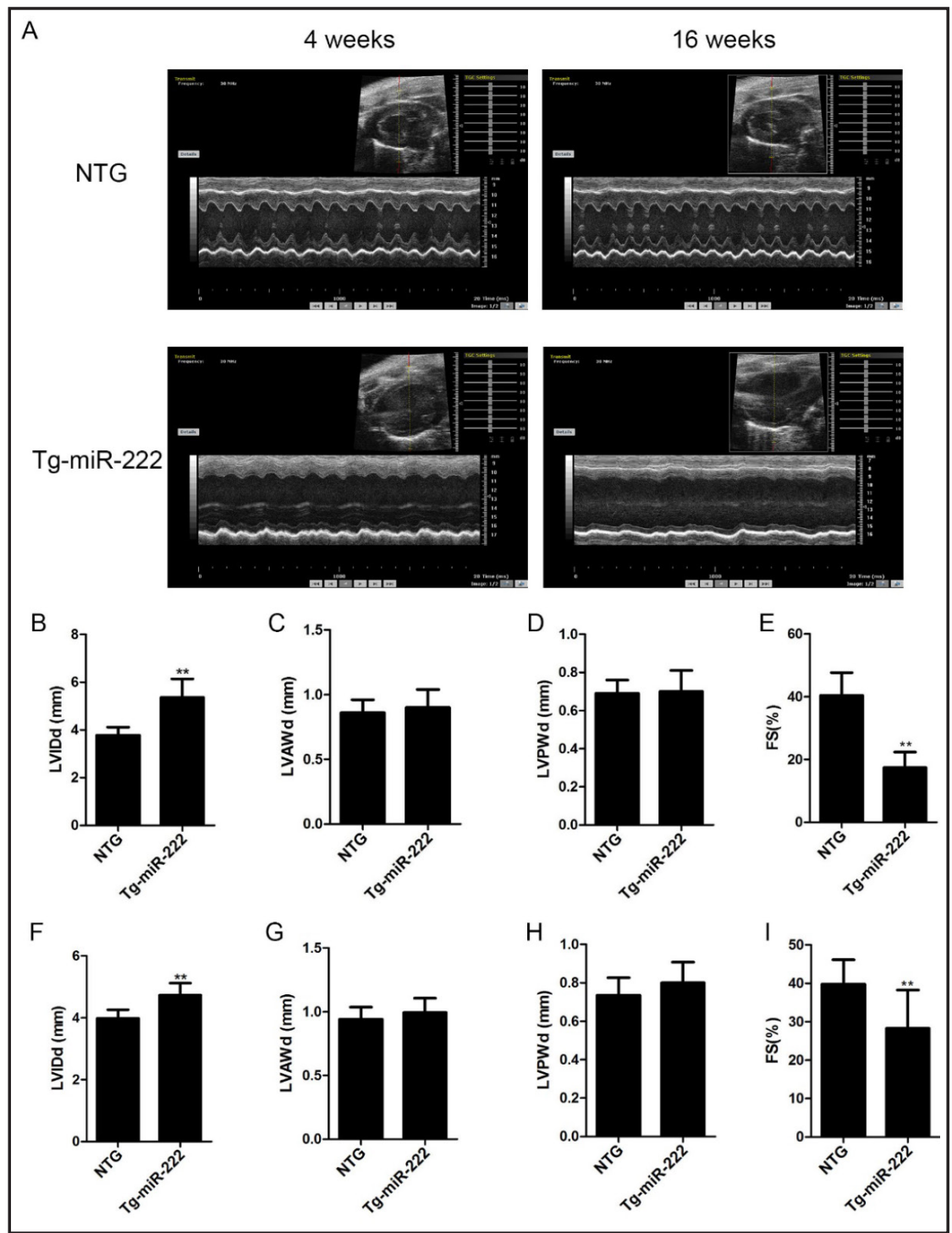

miR-222 induces cardiac fibrosis and apoptosis in vivo

Cardiac fibrosis and apoptosis are important pathological changes in cardiac remodeling. Using Masson's trichrome staining, we observed an excessive deposition of collagen in the myocardia of Tg-miR-222 mice (Fig. 3A and B). Moreover, the number of TUNEL-positive cells was increased in the myocardial tissues of Tg-miR-222 mice, which indicated that constitutive overexpression of miR-222 in cardiomyocytes could induce apoptosis in vivo (Fig. 3C and D). These results indicated that overexpression of miR-222 resulted in a severe cardiac fibrosis and a diminished number of cardiomyocytes, which may be directly associated with the impairment of cardiac function in Tg-miR-222 mice.

miR-222 downregulates $p 27$ in cardiomyocytes

Previously, our results indicated that miR-221 could induce cardiac remodeling through targeting $\mathrm{p} 27$ expression. Because $\mathrm{p} 27$ was also predicted as a target of miR-222, we therefore detected the expression of p27 in NRCM transfected with the miR-222 mimic. We observed that the expression of p27 was dramatically downregulated in miR-222-transfected NRCM (Fig. 4A and B). Accordingly, the expression of p27 was downregulated in the hearts of TgmiR-222 mice (Fig. 4C and D), indicating that p27 could be downregulated by miR-222, both in vitro and in vivo. 
Fig. 3. Cardiac-specific overexpression of miR-222 results in cardiac fibrosis and apoptosis. (A) Masson's trichrome-stained heart tissues from miR-222 transgenic mice (TgmiR-222) and the non-transgenic controls (NTG). Scale bars: $20 \mu \mathrm{m}$. (B) Collagen deposition from NTG and Tg-miR-222 hearts $(n=5$ per group). (C) TUNEL labeling of nuclei in hearts from NTG and Tg-miR-222 mice. Scale bars: $20 \mu \mathrm{m}$. (D) The percentages of TUNEL-positive cells in hearts from NTG and Tg-miR-222 mice ( $n=5$ per group).

Fig. 4. miR-222 downregulates p27 in cardiomyocytes. (A, B) Neonatal rat cardiomyocytes were transfected with either a miR-222 mimic or scrambled RNA as a negative control (A). The expression of p27 was measured by Western blotting, and the relative expression is shown (B). (C, D) The expression of p27 in hearts from miR-222 transgenic mice (Tg-miR-222) and the non-transgenic controls (NTG). ${ }^{*} \mathrm{P}<0.05$ and ${ }^{* *} \mathrm{P}<0.01$ compared with the control group ( $n=3$ per group).



A

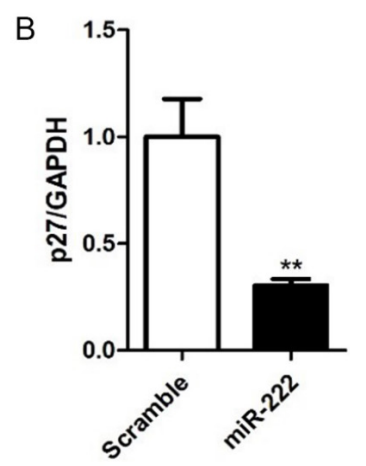

C

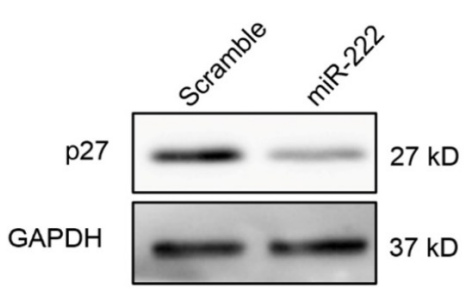

。

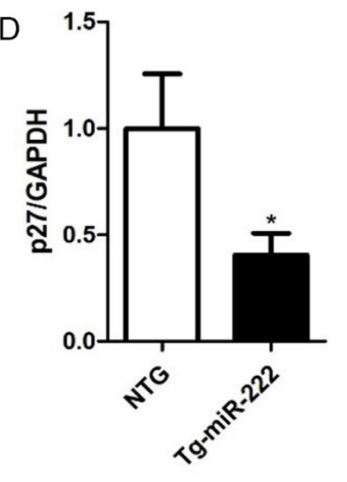

miR-222 inhibits autophagy in miR-222 transgenic mice

Given that miR-221 inhibited autophagy in cardiomyocytes by modulating the p27/ CDK2/mTOR axis [3], we hypothesized that miR-222 might also regulate autophagy in cardiomyocytes. We detected the expression of autophagy markers, LC3 and p62, in the heart tissues of Tg-miR-222 mice and their NTG littermates. We observed that the expression of LC3-II was downregulated while that of p62 was upregulated in Tg-miR-222 mice (Fig. 5AC). These results indicated that autophagy in the hearts of Tg-miR-222 mice was inhibited.

We then detected the phosphorylation of the mTOR pathway, which is a key regulator of autophagy. We observed that the mTOR pathway was significantly activated in the heart 
A

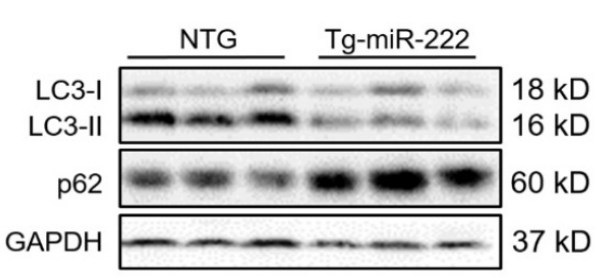

D

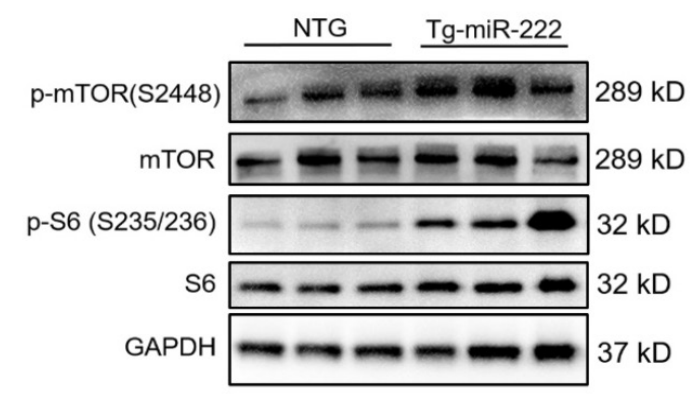



$\mathrm{E}$



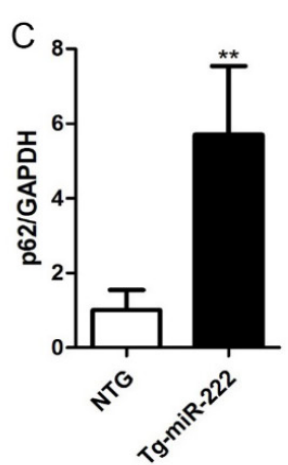

$\mathrm{F}$

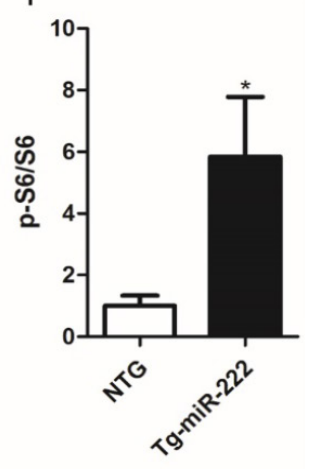

Fig. 5. miR-222 inhibits autophagy in the heart. (A-C) Markers of autophagy (LC3 and p62) in the hearts of miR-222 transgenic mice (Tg-miR-222) and non-transgenic controls (NTG) were determined by Western blotting. The ratios of LC3-II/GAPDH (B) and p62/GAPDH are shown. (D-F) The phosphorylation status of mTOR and S6 was detected in hearts from Tg-miR-222 and NTG mice, respectively. GAPDH was used as an internal control. The phosphorylated/total protein ratios of mTOR (E) and S6 (F) are shown. ${ }^{*} \mathrm{P}<0.05$ and $* * \mathrm{P}<0.01$ compared with the NTG group ( $\mathrm{n}=3$ per group).

tissues of Tg-miR-222 mice, as shown by the increased phosphorylation of mTOR and S6 (Fig. 5D-F). Taken together, our data demonstrated that miR-222 inhibited autophagy in cardiomyocytes and may be associated with the downregulation of p27 and the activation of mTOR pathway.

\section{Discussion}

The present study uncovered the role of miR-222 in the regulation of pathological cardiac remodeling. We found that cardiac-specific overexpression of miR-222 resulted in significant heart failure. Moreover, miR-222 downregulated the expression of p27 and mediated the regulation of autophagy in the heart. Our results indicated that miR-222 participated in the regulation of pathological cardiac remodeling and heart failure.

Pathological cardiac remodeling is a chronic process that is orchestrated by a group of cardiac-associated miRNAs. The roles of these miRNAs may vary according to the context or phase of cardiac remodeling. In exercise-induced cardiac growth, the upregulation of miR222 promoted cell proliferation [14]. These effects were mediated by targeting HIPK1 and HMBOX1 [14]. In contrast, we observed that cardiac-specific overexpression of miR-222 in vivo induced a severe impairment in cardiac function, as indicated by increased ANP and BNP expression, cardiac fibrosis, and apoptosis in the heart tissues; all of which, suggest cardiac remodeling.

In miR-221 transgenic mice, the downregulation of p27 contributed to the inhibition of pathological cardiac remodeling and heart failure [3]. In angiotensin II-induced cardiac 


\section{Cellular Physiology Cell Physiol Biochem 2016;39:1503-1511 \begin{tabular}{ll|l|l} 
DOI: 10.1159/000447853 & $\begin{array}{l}\text { O 2016 The Author(s). Published by S. Karger AG, Basel } \\
\text { www.karger.com/cpb }\end{array}$ \\
\hline
\end{tabular} \\ Su et al.: miR-222 Induces Heart Failure}

remodeling models, an increase of p27 protects against cardiac hypertrophy [5, 6]. Previous reports suggest that p27 is a bona fide target gene of miR-222 in various cell lines [8-12]. Our data revealed that $\mathrm{p} 27$ was downregulated by miR-222 overexpression in cardiomyocytes, both in vitro and in vivo, which indicated that downregulation of p27 may be involved in miR222-induced cardiac remodeling and heart failure.

Autophagy is an important process that maintains normal cardiomyocyte function, whereas uncontrolled autophagy leads to cardiac remodeling [16-22]. Autophagy inhibition impairs the cardiac function, which is associated with decreased clearance of protein aggregates, deteriorating injured organelles [23]. Blunting of autophagy by inhibit Atg5 expression induced an impairment of cardiac function [16]. In Tg-miR-222 mice, we also observed a significant inhibition of autophagy, as evidenced by the decrease of LC3II and increase of p62. These changes of autophagy induced by miR-222 may result in an accumulation of protein aggregates and injured organelles, which may be associated with the impairment of cardiac function.

The activation of autophagy is precisely regulated by various signaling pathways. The p27 and mTOR signaling pathway play pivotal roles in regulating the level of autophagy. In our previous study, we found that miR-221 suppresses autophagy by directly targeting p27, and the autophagy impairment is indispensable to miR-221-induced heart failure [3]. It has been well known that the miR-222 and miR-221 locate in the same gene cluster, and their seed sequences are identical. These two miRNAs share many target genes. By targeting p27, miR-221 inhibited cardiac autophagy by modulating the mTOR axis [3]. In the present study, we found that miR-222 inhibited p27 and resulted in cardiac dysfunction and autophagy impairment, same as what miR-221 did. miR-222 overexpression induced the activation of the mTOR pathway. The role of miR-222 in the regulation of mTOR activation is not understood, however, the downregulation of p27 may be involved. The robust expression of p27 can protect stressed adult hearts by activating autophagy to avoid apoptosis [7]. Consistently, our data showed that apoptosis in the hearts of Tg-miR-222 mice was increased, supporting that the downregulation of $\mathrm{p} 27$ and autophagy inhibition may be associated with the pathological cardiac remodeling and heart failure induced by miR-222 overexpression.

Our study revealed that cardiac-specific overexpression of miR-222 could induce pathological cardiac remodeling and heart failure, which may be associated with the p27/ mTOR/autophagy axis. Our results suggest that miR-222 may be a potential therapeutic target for cardiac remodeling and heart failure.

\section{Acknowledgements}

This study was supported by the China Postdoctoral Science Foundation (2015M581025) and the National Science Foundation of China (81500236 and 81570270).

\section{Disclosure Statement}

The authors declare no competing financial interests.

\section{References}

1 Valencia-Sanchez MA, Liu J, Hannon GJ, Parker R: Control of translation and mrna degradation by mirnas and sirnas. Genes Dev 2006;20:515-524.

2 Wang C, Wang S, Zhao P, Wang X, Wang J, Wang Y, Song L, Zou Y, Hui R: Mir-221 promotes cardiac hypertrophy in vitro through the modulation of p27 expression. J Cell Biochem 2012;113:2040-2046.

3 Su M, Wang J, Wang C, Wang X, Dong W, Qiu W, Wang Y, Zhao X, Zou Y, Song L, Zhang L, Hui R: Microrna-221 inhibits autophagy and promotes heart failure by modulating the $\mathrm{p} 27 / \mathrm{cdk} 2 / \mathrm{mtor}$ axis. Cell Death Differ 2015;22:986-999. 


\section{Cellular Physiology Cell Physiol Biochem 2016;39:1503-1511 and Biochemistry Published online: September 12, $2016 \begin{aligned} & \text { DOI: 10.1159/000447853 } 2016 \text { The Author(s). Published by S. Karger AG, Basel } \\ & \text { www.karger.com/cpb }\end{aligned}$ \\ Su et al.: miR-222 Induces Heart Failure}

4 Konecny F, Zou J, Husain M, von Harsdorf R: Post-myocardial infarct p27 fusion protein intravenous delivery averts adverse remodelling and improves heart function and survival in rodents. Cardiovasc Res 2012;94:492-500.

$5 \quad$ Li J, Zhang C, Xing Y, Janicki JS, Yamamoto M, Wang XL, Tang DQ Cui T: Up-regulation of p27(kip1) contributes to nrf2-mediated protection against angiotensin ii-induced cardiac hypertrophy. Cardiovasc Res 2011;90:315-324.

6 Hauck L, Harms C, An J, Rohne J, Gertz K, Dietz R, Endres M, von Harsdorf R: Protein kinase ck2 links extracellular growth factor signaling with the control of p27(kip1) stability in the heart. Nat Med 2008;14:315-324.

7 Sun X, Momen A, Wu J, Noyan H, Li R, von Harsdorf R, Husain M: P27 protein protects metabolically stressed cardiomyocytes from apoptosis by promoting autophagy. J Biol Chem 2014;289:16924-16935.

8 Zhong C, Ding S, Xu Y, Huang H: Microrna-222 promotes human non-small cell lung cancer h460 growth by targeting p27. Int J Clin Exp Med 2015;8:5534-5540.

9 Yang YF, Wang F, Xiao JJ, Song Y, Zhao YY, Cao Y, Bei YH, Yang CQ: Mir-222 overexpression promotes proliferation of human hepatocellular carcinoma hepg2 cells by downregulating p27. Int J Clin Exp Med 2014;7:893-902.

10 Sun C, Li N, Zhou B, Yang Z, Ding D, Weng D, Meng L, Wang S, Zhou J, Ma D, Chen G: Mir-222 is upregulated in epithelial ovarian cancer and promotes cell proliferation by downregulating p27. Oncol Lett 2013;6:507512.

11 Hu L, Ibrahim S, Liu C, Skaar J, Pagano M, Karpatkin S: Thrombin induces tumor cell cycle activation and spontaneous growth by down-regulation of p27kip1, in association with the up-regulation of skp2 and mir-222. Cancer Res 2009;69:3374-3381.

12 Miller TE, Ghoshal K, Ramaswamy B, Roy S, Datta J, Shapiro CL, Jacob S, Majumder S: Microrna-221/222 confers tamoxifen resistance in breast cancer by targeting p27kip1. J Biol Chem 2008;283:29897-29903.

13 Frenquelli M, Muzio M, Scielzo C, Fazi C, Scarfo L, Rossi C, Ferrari G, Ghia P, Caligaris-Cappio F: Microrna and proliferation control in chronic lymphocytic leukemia: Functional relationship between mir-221/222 cluster and p27. Blood 2010;115:3949-3959.

14 Liu X, Xiao J, Zhu H, Wei X, Platt C, Damilano F, Xiao C, Bezzerides V, Bostrom P, Che L, Zhang C, Spiegelman $\mathrm{BM}$, Rosenzweig A: Mir-222 is necessary for exercise-induced cardiac growth and protects against pathological cardiac remodeling. Cell Metab 2015;21:584-595.

15 Wang X, Wang J, Su M, Wang C, Chen J, Wang H, Song L, Zou Y, Zhang L, Zhang Y, Hui R: Tnni3k, a cardiacspecific kinase, promotes physiological cardiac hypertrophy in transgenic mice. PLoS One 2013;8:e58570.

16 Taneike M, Yamaguchi O, Nakai A, Hikoso S, Takeda T, Mizote I, Oka T, Tamai T, Oyabu J, Murakawa T, Nishida K, Shimizu T, Hori M, Komuro I, Takuji Shirasawa TS, Mizushima N, Otsu K: Inhibition of autophagy in the heart induces age-related cardiomyopathy. Autophagy 2010;6:600-606.

17 Ceylan-Isik AF, Dong M, Zhang Y, Dong F, Turdi S, Nair S, Yanagisawa M, Ren J: Cardiomyocyte-specific deletion of endothelin receptor a rescues aging-associated cardiac hypertrophy and contractile dysfunction: Role of autophagy. Basic Res Cardiol 2013;108:335.

18 Thomas RL, Roberts DJ, Kubli DA, Lee Y, Quinsay MN, Owens JB, Fischer KM, Sussman MA, Miyamoto S, Gustafsson AB: Loss of mcl-1 leads to impaired autophagy and rapid development of heart failure. Genes Dev 2013;27:1365-1377.

19 Hu P, Zhou H, Lu M, Dou L, Bo G, Wu J, Huang S: Autophagy Plays a Protective Role in Advanced Glycation End Product-Induced Apoptosis in Cardiomyocytes. Cell Physiol Biochem 2015;37:697-706.

20 Liu L, Wang C, Sun D, Jiang S, Li H, Zhang W, Zhao Y, Xi Y, Shi S, Lu F, Tian Y, Xu C, Wang L: Calhex 231 Ameliorates Cardiac Hypertrophy by Inhibiting Cellular Autophagy in Vivo and in Vitro. Cell Physiol Biochem 2015;36:1597-1612.

21 Hsieh DJ, Kuo WW, Lai YP, Shibu MA, Shen CY, Pai P, Yeh YL, Lin JY, Viswanadha VP, Huang CY: 17 $\beta$-Estradiol and/or Estrogen Receptor $\beta$ Attenuate the Autophagic and Apoptotic Effects Induced by Prolonged Hypoxia Through HIF-1 $\alpha$-Mediated BNIP3 and IGFBP-3 Signaling Blockage. Cell Physiol Biochem 2015;36:274-284.

22 Wang R, Liu YY, Liu XY, Jia SW, Zhao J, Cui D, Wang L: Resveratrol protects neurons and the myocardium by reducing oxidative stress and ameliorating mitochondria damage in a cerebral ischemia rat model. Cell Physiol Biochem 2014;34:854-864.

23 Linton PJ, Gurney M, Sengstock D, Mentzer RM Jr, Gottlieb RA: This old heart: Cardiac aging and autophagy. J Mol Cell Cardiol 2015;83:44-54. 